\section{Herausforderung regionale Nachhaltigkeit}

\author{
Regionalwirtschaftliche Ansätze als Konzept zur nachhaltigen Entwicklung sto- \\ Ben in den letzten Jahren auf hohes Interesse. Viele Aspekte nachhaltigen regi- \\ onalen Wirtschaftens sind jedoch noch ungeklärt. Drei Kernprobleme sind die \\ noch zu lösenden Organisationsfragen, die Frage einer generellen Bevorzugung \\ von regionalen Kreisläufen und die notwendigen politischen Förderungen. Diese \\ werden anhand ausgewählter Praxiserfahrungen diskutiert.
}

A

Von Dirk Günther

1 Ebene werden Regionen zunehmend als eine wichtige Säule bei der Umsetzung einer nachhaltigen Entwicklung gesehen. Regionen sind kleinräumig genug, um überschaubar zu sein auch wenn sie nicht notwendigerweise geographisch definiert sein müssen. Auch können Ursachen und Wirkungen von wirtschaftlichen Handlungen unmittelbar erfahren und relativ gut zugeordnet werden, regionale Akteure agieren häufig in regionalen Netzwerken und sind miteinander persönlich bekannt. All dies kommt der Umsetzung eines nachhaltigen Wirtschaftens entgegen. Viele Aspekte sind jedoch noch offen: Inwieweit sind etwa regionale Kreisläufe überhaupt sinnvoll? Wie können sich selbst tragende Strukturen, die das Human- und Naturpotenzial einer Region nutzen, entwickeln? Wie lassen sich Netzwerke von Unternehmen zur gegenseitigen Ressourcennutzung bilden, wie Wettbewerb und Nachhaltigkeit verbinden? Einzelne Erfahrungen aus Pilotprojekten liegen zu diesen Fragen schon vor (1).

Das Bundesministerium für Bildung und Forschung (BMBF) hat mit seiner Förderinitiative "Modellprojekte für nachhaltiges Wirtschaften. Regionale Ansätze nachhaltigen Wirtschaftens" seit 1998 mit 15 Modellprojekten versucht, zur Klärung dieser Fragen beizutragen, neue Denkansätze in Forschung und Praxis zu schaffen und das Potenzial der regionalen Ebene zur Umsetzung des Leitbildes Nachhaltige Entwicklung abzuschätzen (2).

Zum Abschluss der Initiative führte der Internationale Rat für lokale Umweltinitiativen (ICLEI) im November letzten Jahres in Leipzig im Auftrag des BMBF eine internationale Konferenz zum Thema nachhaltiges regionales Wirtschaften durch (3).
Als anschauliches und zentrales Ergebnis haben die Teilnehmer acht Hypothesen zu regionalem Wirtschaften erarbeitet, die auch dem Ziel der Förderung nachhaltigen regionalen Wirtschaftens und der nachhaltigen Regionalentwicklung dienen sollen, in dem sie die gemeinsame Basis für die Etablierung internationaler Forschungs- und Umsetzungskooperationen darstellen (4). Hieraus können drei Kernforderungen für regionale Ansätze nachhaltigen Wirtschaftens herausgestellt werden:

1. Die anstehenden Aufgaben und noch zu lösenden Organisationsfragen,

2. die Frage nach der generellen Bevorzugung von regionalen Kreisläufen und

3. die notwendigen politischen Förderungen.

Die Grundlage bildet dabei ein Begriffsverständnis, das eine Region nicht als eine politisch verfasste territoriale Einheit begreift. Vielmehr werden unter Region sowohl geographische Gebiete als auch Zusammenschlüsse oder Institutionen einer Größe, die irgendwo zwischen Kommune und Nationalstaat liegt, verstanden. Diese können innerhalb eines Staates oder aber länderübergreifend angesiedelt sein und definieren sich über ein Geflecht von wirtschaftlichen, sozialen oder ökologischen Beziehungen.

\section{Regionale Kooperation organisieren}

Sowohl in den Modellprojekten der Förderinitiative als auch in vielen anderen Forschungs- und Praxisprojekten hat sich herausgestellt, dass Kooperationen und Netzwerke regionaler Akteure eine wichtige Rolle einnehmen. Trotz des inzwischen beträchtlichen Erfahrungsschatzes auf diesem Gebiet sind noch viele Fragen zum Thema "Wie funktioniert regionale Kooperation?" offen.
So müssen funktionsfähige Ansätze gefunden werden zur Ermittlung derjenigen Regionalkonzepte, die allen Aspekten nachhaltiger Entwicklung gerecht werden.

Als entscheidend für die Erfüllung des Anspruchs der Nachhaltigkeit hat sich erwiesen, dass regionale Entwicklung auf dem Prinzip der Partizipation aufbaut und eine Vielzahl von Akteuren einschließt. So hat beispielsweise das BMBF-Modellprojekt "Zukunft der Arbeit und nachhaltiges regionales Wirtschaften" der Stiftung Bauhaus Dessau für eine altindustrielle Region Ostdeutschlands unter Beteiligung der relevanten Akteure erfolgreich ein neues Regionalkonzept auf der Basis der Nachhaltigkeit entwickelt (5). Derselbe Ansatz wurde in der Raumplanung vom Ecoinstitut Barcelona in Spanien für die Reaktivierung des Barcelonaer Stadtteils Trinitat Nova verfolgt (6).

Sofern man auf die Beteiligung von Unternehmen angewiesen ist, bedarf es auch neuer Kooperationsmodelle, die ohne die Preisgabe wettbewerbskritischer Unternehmensdaten auskommen, effektiver Wege zu einer Kooperation im Sinne regionaler Innovation (Lernen lernen), neuer Lösungen für regionale Logistik- und Produktionsfragen sowie Ansätze zur kommunalen Beteiligung auf regionaler Ebene.

Entscheidend ist ein Klima gegenseitigen Vertrauens der an der Kooperation teilnehmenden Akteure. Dies zeigte sich deutlich im BMBF-Modellprojekt des Instituts für Umweltwirtschaftsanalysen Heidelberg (IUWA), das sich mit regionalen Stoffkreisläufen und -management in der Region Rhein-Neckar auseinandergesetzt hat (7).

Es kann hier sicherlich kein Patentrezept im Sinne einer Blaupause für jede Region geben. Jede Region muss ihr eigens Konzept unter Berïcksichtigung der jeweiligen Gegebenheiten entwickeln. Doch können Regionen auch von einander lernen. Positive Beispiele und Erfahrungen sind in Europa vorhanden. Denn, um das Rad nicht immerzu neu erfinden zu müssen, sollten Erfahrungen mit regionaler Kooperation stets weitergegeben werden. Dies lässt sich am einfachsten über nationale und internationale Akteursnetze erreichen.

\section{Regionale Kreisläufe und Handel}

Eine weitere spannende Diskussion gibt es um die Frage, ob regionale Kreisläufe grundsätzlich nachhaltiger sind. Eine Vielzahl der auf der Konferenz vorgestellten Projekte haben sich mit der Untersuchung bzw. Umsetzung regionaler Stoffkreisläufe aus unterschiedlichen Blickwinkeln 
beschäftigt. Es kann eindeutig festgehalten werden, dass regionale Kooperation und überregionaler Handel sich nicht gegenseitig ausschließen. Im Gegenteil, sie bedingen sich oft. So kann Zusammenarbeit in der Region einerseits einen Teil der überregionalen Handelsaktivitäten ersetzen und wird andererseits auch den Wettbewerb exportorientierter Branchen anregen. Gerade für regionsgrenznahe Gebiete kann sich aber auch grenzübergreifender gegenüber innerregionalem Handel als sinnvollere Alternative erweisen.

Zwar ist es sowohl ökonomisch als auch ökologisch sinnvoll, Transportwege zu vermeiden, wenn ihr Anteil an den Produktkosten den Großteil ausmacht, und statt dessen Handelsbeziehungen zu einer zentralen bzw. geographisch günstiger gelegenen Produktionsstätte aufzubauen. Aus diesem Grunde ist es wichtig, dass Transportpreise in Europa die externen Kosten des Transports, die auch durch die Wahl des Transportmittels beeinflusst werden, in zunehmendem Maße widerspiegeln. Abgeschafft werden sollten auch Subventionen, die nur auf Nachweis der exportwirtschaftlichen Relevanz gewährt werden. Hier ist insbesondere die europäische, aber auch die nationale Regionalförderung angesprochen. Andererseits ist ein regionales Stoffstrommanagement zwischen Unternehmen nicht einfach zu installieren. Von der regionalen Verfügbarkeit bis hin zu Vertrauensfragen sind viele Hindernisse zu überwinden (8). Zudem geraten regionale Stoffkreisläufe, die mit dem Anspruch der Nachhaltigkeit gestartet sind, schnell in Gefahr diese zu vergessen, wenn sie unter Wettbewerbsdruck stehen. Viele Projekte vor allem im landwirtschaftlichen Bereich haben gezeigt, dass eine Balance zwischen den drei Säulen der Nachhaltigkeit schwierig ist, wenn eine marktwirtschaftliche Strategie verfolgt wird.

Letztlich entsteht, auch ökologisch betrachtet, die Nachhaltigkeit nicht aus der Herkunft des Produkts, sondern aus den Produkt- und Prozessstandards mit denen es produziert wurde. Regionale Kreisläufe können hier ein höheres Maß an Transparenz bewirken.

\section{- Öffentliche Unterstützung von Innovationen}

Ein wichtiges Standbein der nachhaltigen Entwicklung liegt in der Verbesserung regionaler Systeme, insbesondere des Innovationssystems. Dabei kommt insbesondere der Verstetigung der Kooperation im Sinne einer zielgerichteten systemischen Integration der Akteure und der Bildung von Lerngemeinschaften eine wichtige Bedeutung zu. So wird das regionale System zusehends eigenständiger und stabiler.

Bei der Betrachtung von regionalen Innovationssystemen ist aber nicht nur an technologische Innovationen zu denken. Innovationen im Rahmen regionaler Kooperation, das heißt institutionelle Innovationen, sind ein integraler Bestandteil jeder Innovationslandschaft und stehen den technologischen in ihrer Wichtigkeit nicht nach. Die entscheidende Rolle des Aufbaus institutioneller Kapazitäten für diesen Prozess kommt internationalen Institutionen, nationalen Regierungen und regionalen Institutionen gleichermaßen zu. Doch wie alle Innovationsaktivitäten verdient auch regionale Innovation nur dann öffentliche Förderung, wenn sie zur Nachhaltigkeit in dem Sinne beiträgt, dass sie mindestens einen ihrer Aspekte stärkt und dies nicht zu Lasten der jeweils anderen geht. Für die Regionalförderung könnte dies als Orientierungshilfe dienen.

Interregionale Kooperation und Vernetzung dürfen nicht von nationalen und internationalen Wirtschafts- und Gesetzesrichtlinien behindert werden. Vielmehr sollten Kooperationen und gegenseitige Lernprozesse gefördert werden. In diesem Sinne ist eine Zusammenarbeit der verschiedenen Regierungsinstitutionen erforderlich. Ein entscheidender Bestandteil der Förderung ist dabei eine Vergrößerung des Einflusses und des Budgetanteils speziell kommunaler sowie regionaler Verwaltungsebenen. Lokal und regional lassen sich Lernprozesse und Kooperationen wesentlich besser und effektiver initiieren und fördern, als dies nationalstaatlich möglich wäre. Dies haben zahlreiche Modellprojekte anschaulich gemacht. Auch in der nationalen und internationalen Gesetzesund Richtliniensetzung muss dies berïcksichtigt werden. Aktuell stellt sich diese Herausforderung im Rahmen der Neuverhandlungen der verschiedenen Abkommen bei der Welthandelsorganisation (WTO), beispielsweise des Generell Agreements on Trade in Services (GATS) (9).

\section{- Schlussfolgerungen}

Nachhaltiges Wirtschaften lässt sich in regionalen Zusammenhängen sehr gut und erfolgreich umsetzen. Dies haben die Modellprojekte der Förderinitiative des BMBF gezeigt und wurde von den vorgestellten Projekten der Regional Cycles Konferenz bestätigt. Die Projekte haben aber auch gezeigt, dass diese Umsetzung sich nicht einfach gestaltet. Viele Hemmnisse, Hürden und Fehlentwicklungen stehen dem Konzept entgegen.
Alle Projekte haben gezeigt, dass es entscheidend auf die Schlüssigkeit eines regionalen Konzepts ankommt, das Kooperationen fördert, Vertrauen schafft und vor allem politisch und institutionell unterstützt und gefördert wird. Die Modellprojekte in ganz Europa haben hier einen wichtigen Schritt getan, der aber für eine breite, sich selbst tragende Umsetzung nicht ausreichend ist. Weitere Forschung und Modellumsetzung, und das heißt insbesondere Förderung, ist hier nötig.

\section{Anmerkungen}

(1) Vgl. Kluge, T./ Schramm, E. (Hrsg.): Regional Approaches to Sustainable Economy: Potentials and Limits. Experiences from German Case-Studies. ISOE, MSÖ 19, Frankfurt am Main 2002. Vgl. zum Thema auch die Schwerpunkte 1/2002 und 5/1998 von Ökologisches Wirtschaften.

(2) Vgl. gsf: Innovative Ansätze zur Stärkung der regionalen Ökonomie. Föderinitiative Modellprojekte für nachhaltiges Wirtschaften 1998-2002; gsf/ BMBF, München 1999. Zusammenfassungen aller 15 Modellprojekte sind unter www.iclei.org/economy/bmbf.htm verfügbar. (3) Unter dem Titel "Regionale Kreisläufe: Regionales Wirtschaften auf dem Weg zur Nachhaltigkeit" widmeten sich internationale Experten, insbesondere aus Kommunen, Regionalverwaltungen und Vertreter nationaler Einrichtungen, sowie Forscher, Entwickler und Anwender, Fragen zu Themen nachhaltiger Entwicklung und regionales Wirtschaften. Nähere Informationen unter www.iclei.org/economy/ conference

(4) Vgl. ICLEl: Regional Cycles - Regional Economy Towards Sustainability. Conference Report, ICLEl, Freiburg (im Erscheinen).

(5) Für nähere Informationen: www.nachhaltig.org/ bauhaus/reg04fr.htm

(6) Für nähere Informationen: www.pangea.org/trinova/ documentos/easwf.doc

(7) Nähere Informationen unter www.nachhaltig.org/ iuwa/reg06fr.htm

(8) Vgl. hierzu Graehl, S. et al: Konzepte für ein überbetriebliches regionales Stoffstrommanagement. In: Ökologisches Wirtschaften, Nr. 6/2000, S. 31-33.

(9) Vgl. ICLEl: Sustainable regions and cities under new global rules. Survey on the General Agreement on Trade in Services and the Johannesburg process, ICLEl, Freiburg 2003.

\section{Der Autor}

Dirk Günther ist Sozialwissenschaftler und arbeitete bis Februar 2003 als Projektmitarbeiter im Bereich Umweltfreundliche Beschaffung und Öko-effizientes Wirtschaften des Städtenetzwerks ICLEI. Kontakt: Dirk Günther, Tel. 0761-7691257, E-Mail: d_guenther@web.de 
(c) 20I0 Authors; licensee IÖW and oekom verlag. This is an article distributed under the terms of the Creative Commons Attribution Non-Commercial No Derivates License (http://creativecommons.org/licenses/by-nc-nd/3.o/), which permits unrestricted use, distribution, and reproduction in any medium, provided the original work is properly cited. 